\title{
Sevoflurane induces cardioprotection through reactive oxygen species-mediated upregulation of autophagy in isolated guinea
}

\section{pig hearts.}

\author{
Mayumi Shiomi \\ Osaka Medical College, Takatsuki, Japan \\ Masami Miyamae \\ Osaka Dental University, Osaka, Japan
}

Genzou Takemura

Gifu University Graduate School of Medicine, Gifu, Japan

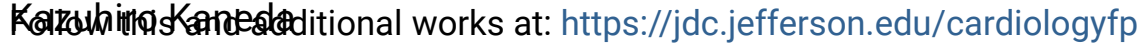 \\ zi stein Medical Center and Jefferson Medical College \\ Part of the Cardiology Commons \\ kelltitakg Ikamona how access to this document benefits you Einstein Medical Center and Jefferson Medical College}

\section{Recommended Citation}

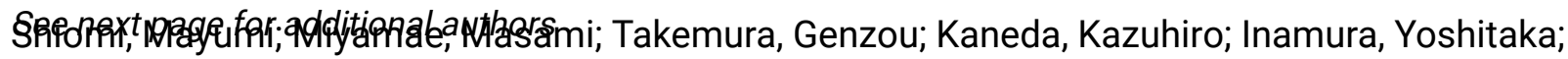

Onishi, Anna; Koshinuma, Shizuka; Momota, Yoshihiro; Minami, Toshiaki; and Figueredo, Vincent M., "Sevoflurane induces cardioprotection through reactive oxygen species-mediated upregulation of autophagy in isolated guinea pig hearts." (2013). Division of Cardiology Faculty Papers. Paper 32.

https://jdc.jefferson.edu/cardiologyfp/32

This Article is brought to you for free and open access by the Jefferson Digital Commons. The Jefferson Digital Commons is a service of Thomas Jefferson University's Center for Teaching and Learning (CTL). The Commons is a showcase for Jefferson books and journals, peer-reviewed scholarly publications, unique historical collections from the University archives, and teaching tools. The Jefferson Digital Commons allows researchers and interested readers anywhere in the world to learn about and keep up to date with Jefferson scholarship. This article has been accepted for inclusion in Division of Cardiology Faculty Papers by an authorized administrator of the Jefferson Digital Commons. For more information, please contact: JeffersonDigitalCommons@jefferson.edu. 


\section{Authors}

Mayumi Shiomi, Masami Miyamae, Genzou Takemura, Kazuhiro Kaneda, Yoshitaka Inamura, Anna Onishi, Shizuka Koshinuma, Yoshihiro Momota, Toshiaki Minami, and Vincent M. Figueredo 


\title{
As submitted to:
}

\author{
Journal of Anesthesia
}

\section{And later published as:}

\section{Sevoflurane Induces Cardioprotection Through Reactive Oxygen Species-Mediated Upregulation of Autophagy in Isolated Guinea Pig Hearts}

\section{December 2013}

Mayumi Shiomi ${ }^{1}$, Masami Miyamae ${ }^{2}$, Genzou Takemura ${ }^{3}$, Kazuhiro Kaneda ${ }^{4}$, Yoshitaka ${ }^{\text {Inamura }}{ }^{4}$ Anna Onishi ${ }^{4}$, Shizuka Koshinuma , Yoshihiro Momota , Toshiaki Minami ${ }^{4}$, Vincent M. Figueredo ${ }^{5}$

${ }^{1}$ Department of Anesthesiology, Osaka Medical College, Takatsuki, Japan

${ }^{2}$ Departments of Internal Medicine and ${ }^{4}$ Anesthesiology, Osaka Dental University, Osaka, Japan,

${ }^{3}$ Department of Cardiology, Gifu University Graduate School of Medicine, Gifu, Japan ${ }^{5}$ Institute for Heart and Vascular Health, Einstein Medical Center, and Jefferson Medical College, Philadelphia, USA

Conflict of Interest: The authors have no conflicts of interest to report.

Key Words: sevoflurane, preconditioning, autophagy, ischemia-reperfusion, reactive oxygen species

Corresponding Author \& Reprints: Masami Miyamae, MD, PhD 
Department of Internal Medicine,

Osaka Dental University

8-1 Kuzuha hanazono-cho Hirakata,

Osaka 573-1121, Japan

TEL: 81-72-864-3079, FAX: 81-72-864-3179

E-mail: miyamae0907@gmail.com

Running head: sevoflurane cardioprotection by autophagy

Word count: 2480

Number of tables: 1

Number of figures: 4 


\begin{abstract}
Purpose Sevoflurane increases reactive oxygen species (ROS) which mediate cardioprotection against myocardial ischemia-reperfusion injury. Emerging evidence suggests that autophagy is involved in cardioprotection. We examined whether reactive oxygen species mediate sevoflurane preconditioning through autophagy.

Methods Isolated guinea pigs hearts were subjected to $30 \mathrm{~min}$ ischemia followed by 120 min reperfusion (control). Anesthetic preconditioning was elicited with $2 \%$ sevoflurane for 10 minutes prior to ischemia (SEVO). The ROS-scavenger, n-(2-mercaptopropionyl) glycine (MPG, 1mmol/L) was administered starting $30 \mathrm{~min}$ before ischemia to sevofluranetreated (SEVO+MPG) or non-sevoflurane-treated (MPG) hearts. Infarct size was determined by triphenyltetrazolium chloride stain. Tissue samples were obtained after reperfusion to determine autophagy-related protein (microtubule-associated protein light chain I and II: LC3-I, II) and 5'AMP-activated protein kinase (AMPK) expression using Western blot analysis. Electron microscopy was used to detect autophagosomes.
\end{abstract}

Results Infarct size was significantly reduced and there were more abundant autophagosomes in SEVO compared with control. Western blot analysis revealed that the ratio of LC3-II/I and phosphorylation of AMPK were significantly increased in SEVO. These effects were abolished by MPG.

Conclusions Sevoflurane induces cardioprotection through ROS-mediated upregulation of autophagy.

180 words 


\section{Introduction}

Sevoflurane is a popular anesthetic with few clinical side effects and is used worldwide. Over the last 15 years, experimental [1-5] and clinical studies [6] have demonstrated that volatile anesthetics, including sevoflurane, exert cardioprotective effects against myocardial ischemia-reperfusion injury such as ischemic preconditioning where brief episodes of ischemia and reperfusion before prolonged ischemia dramatically limit infarct size [7]. Many potential mechanisms for this cardioprotection known as anesthetic preconditioning (APC) have been proposed [8]. Novalija et al. demonstrated that APC by sevoflurane is initiated by reactive oxygen species (ROS) produced during sevoflurane exposure and reduces the burst formation of ROS after ischemia-reperfusion in isolated guinea pig hearts [9]. Recently, cardioprotection by sevoflurane has been shown to be mediated by ROS-induced 5' AMP-activated protein kinase (AMPK) activation which is a well known regulator of cellular energy status [10].

AMPK is a key enzyme for the initiation of autophagy which is a tightly regulated intracellular catabolic process for the disposal of damaged and dysfunctional organelles and protein aggregates to maintain homeostasis [11]. Growing evidence suggests that autophagy is activated during various pathological conditions, including myocardial ischemiareperfusion injury [12]. It has been reported that excessive activation of autophagy can be detrimental and may even lead to cell death called autophagic cell death [13]. In contrast, Sala-Mercado et al. demonstrated that pre-ischemic induction of autophagy limits myocardial infarct size in in vivo swine hearts [14]. Qiao et al. demonstrated that cardioprotection of delayed preconditioning by sevoflurane is mediated by upregulation of autophagy [15]. However, it has not been determined whether activation of autophagy is 
beneficial or detrimental in myocardial ischemia-reperfusion injury. Although ROS mediate sevoflurane preconditioning, it is not known whether autophagy is involved in this ROSinduced cardioprotection. Thus, we hypothesized that ROS produced during sevoflurane exposure mediates sevoflurane cardiac preconditioning through activation of autophagy. Elucidating the role of autophagy in sevoflurane preconditioning could prove clinically useful in patients at cardiovascular risk during perioperative periods. 


\section{Materials and Methods}

This study was conducted in accordance with the Guidelines for Animal Research at Osaka Dental University, and with the approval of the Animal Experiment Committee of Osaka Dental University, Osaka, Japan. These guidelines conform to those laid out in the Guide for the Care and Use of Laboratory Animals, available from the National Academy of Science. Male Hartley guinea pigs were fed Lab Diet (RC4, Oriental Yeast, Tokyo, Japan) and given water ad libitum. A ROS-scavenger, n-(2-mercaptopropionyl) glycine (MPG) was purchased from Sigma Aldrich (Ann Arbor, MI, USA).

Isolated Heart Perfusion and Measurement of Function

Male guinea pigs weighing 550-700g (12-13 weeks old) were given heparin (1000 units intraperitoneally), then anesthetized with pentobarbital $(60 \mathrm{mg} / \mathrm{kg}$, intraperitoneally). Hearts were excised and immediately arrested in cold iso-osmotic saline containing $20 \mathrm{mM}$ $\mathrm{KCl}$. The aorta was cannulated and the isolated hearts were perfused at $70 \mathrm{mmHg}$ on a nonrecirculating isovolumic perfused heart apparatus, using a Krebs-Henseleit perfusate and paced at 240 beats/min as previously described [3]. Left ventricular developed pressure (LVDP; mmHg) was measured using a 2.5 French, high-fidelity micromanometer (NihonKohden, Tokyo, Japan) passed into a compliant latex balloon, inserted into the left ventricle, and recorded on a PowerLab 2/20 Data Recording System (ADInstruments, Hayward, Australia). The balloon was connected to a Y-adapter with one end used to advance the micromanometer and the other used to fill the left ventricular balloon with bubble-free water to an end-diastolic pressure (LVEDP) of $10 \mathrm{mmHg}$. Coronary flow (CF) (ml/min) was measured by collecting effluent. Global ischemia was achieved by clamping the aortic 
inflow line. During ischemia, hearts were maintained at $37^{\circ} \mathrm{C}$ by enclosure in a waterjacketed air chamber. Warmed perfusate kept in the lower part of the chamber saturated the air with humidity and prevented cooling by evaporation. Heart temperature was continuously monitored by a digital thermometer (PTW-100A, Unique Medical, Tokyo, Japan). Sevoflurane was insufflated by passing the $95 \% \mathrm{O}_{2} / 5 \% \mathrm{CO}_{2}$ gas mixture through a calibrated vaporizer (ACOMA, Tokyo, Japan).

Measurement of sevoflurane concentration

Samples of coronary perfusate were collected anaerobically from the aortic cannula for measurement of sevoflurane concentration by an organic vapor sensor (OSP, Saitama, Japan). The organic vapor sensor is designed to detect volatile organic compounds (VOC) in the air. Prior to measurement of sevoflurane concentration, calibration of the organic vapor sensor was performed to make a standard VOC (ppm)-sevoflurane (mmol/L) curve. The concentration of sevoflurane was determined using the well described headspace method [16]. The vial (150ml) containing perfusate obtained from aortic cannula with closed air space in upper portion (headspace) was shaken for one minute. After reaching equilibration in the vial, the organic vapor sensor was connected to the vial to measure VOC (ppm) in the closed air space. The obtained data (ppm) were converted to the concentration of sevoflurane in the perfusate $(\mathrm{mmo} / \mathrm{L})$ using a standard VOC-sevoflurane curve. This concentration correlates well with that measured by the headspace method with gas chromatography which is usually used to measure sevoflurane concentration. 
Experimental Protocol

Animals were assigned to one of 4 groups ( $n=8$ each; Figure 1). After a $20 \mathrm{~min}$ equilibration, baseline LVDP, LVEDP and CF were recorded. Hearts were subjected to 30 $\min$ (control; CTL) of ischemia followed by $120 \mathrm{~min}$ of reperfusion. Anesthetic preconditioning was elicited by administration of sevoflurane (2\%) for $10 \mathrm{~min}$ followed by $10 \mathrm{~min}$ washout before $30 \mathrm{~min}$ (SEVO). A ROS-scavenger, MPG (1 mmol/L) was administered starting $30 \mathrm{~min}$ before ischemia in sevoflurane-treated (SEVO+MPG) or nonsevoflurane-treated animals (MPG) hearts. MPG was dissolved in distilled water and was added to the Krebs-Henseleit perfusate to a final concentration of $1 \mathrm{mmol} / \mathrm{L}$.

Determination of Myocardial Infarct Size

At the end of experiments, hearts were quickly frozen at $-80{ }^{\circ} \mathrm{C}$ for $15 \mathrm{~min}$, and then sliced into $2 \mathrm{~mm}$ thick transverse sections from apex to base (6 slices/heart). After removing the right ventricle and defrosting, each slice was weighed and incubated at $37{ }^{\circ} \mathrm{C}$ with $1 \%$ triphenyltetrazolium chloride (Sigma Chemicals) in phosphate buffer ( $\mathrm{pH} 7.4$ ) for 10 min and then fixed in $10 \%$ formalin for at least $5 \mathrm{~h}$ to distinguish red stained viable tissue from pale unstained necrotic tissue [17]. Each slice was photographed and the necrotic area was determined using Adobe Photoshop ${ }^{\circledR}$ CS (Adobe, San Jose, CA, USA) and multiplied by the weight of the slice, then expressed as a fraction of the left ventricle.

Western Blot Analysis

Separate experiments were performed ( $\mathrm{n}=4$ for each group) to examine expression of autophagy-related protein (microtubule-associated protein light chain I and II: LC3-I, II), and 
AMPK. Myocardial tissue samples were collected after 120min reperfusion, and homogenized in ice-cold homogenizing buffer containing $250 \mathrm{mM}$ sucrose, $20 \mathrm{mM}$ HEPES (pH 7.5), 10 $\mathrm{mM} \mathrm{KCl}, 2 \mathrm{mM}$ EGTA, $2 \mathrm{mM} \mathrm{MgCl}_{2}, 25 \mathrm{mM} \mathrm{NaF}, 50 \mathrm{mM} \beta$-glycerophosphate, $1 \mathrm{mM}$ $\mathrm{Na}_{3} \mathrm{VO}_{4}, 1 \mathrm{mM}$ PMSF and protease inhibitor leupeptin $(10 \mu \mathrm{g} / \mathrm{ml})$. The homogenate was centrifuged at $1000 \mathrm{~g}$ for $5 \mathrm{~min}$ at $4{ }^{\circ} \mathrm{C}$ to clean up. The supernatant was re-centrifuged at $10000 \mathrm{~g}$ for $15 \mathrm{~min}$ at $4{ }^{\circ} \mathrm{C}$ to obtain cytosolic fraction. The protein concentration was estimated with a Bradford assay. Equivalent amounts $(20 \mu \mathrm{g})$ of protein samples were loaded and separated on a $10 \%$ SDS-PAGE gradient gel, then electrically transferred overnight to a polyvinylidene difluoride membrane (Bio-Rad, Hercules, CA, USA). After blocking with 5\% skim milk in Tris-buffered saline containing 0.1\% Tween-20 (TBS-T), the membranes were incubated for $2 \mathrm{~h}$ at $4{ }^{\circ} \mathrm{C}$ in TBS-T containing 5\% skim milk and overnight 1:1000 dilution of rabbit primary antibody for LC3 (Medical \& Biological Laboratories Co LTD, MA, USA), phospho AMPKa (Thr172) and total AMPK (Cell Signaling TECHNOLOGY, Boston, MA, USA). Membranes were incubated with a 1:1000 dilution of horseradish peroxidase-labeled anti-rabbit immunoglobulin G (NA 934V, GE Healthcare, Buckinghamshire, United Kingdom). The same blot was stripped and re-blotted with antibodies to $\alpha$-tubulin (Santa Cruz Biotechnology) (to confirm equal protein loading). Bound antibody signals were detected with enhanced chemiluminescence (Pierce Biotechnology, Rockford, IL) and visualized using VersaDoc 5000 Imaging System (Bio-Rad, Hercules, CA, USA). Quantitative analysis of the band densities was performed by Quantity One software (Bio-Rad), and the results are presented as the ratio of phospho AMPK to total AMPK. The average light intensity was multiplied by 100 to facilitate presentation of an $x$-fold increase.

Electron Microscopy 
To confirm the induction of autophagy, cardiac specimens were obtained at $120 \mathrm{~min}$ of reperfusion ( $\mathrm{n}=4$ for each group). They were fixed with phosphate-buffered $2.5 \%$ glutaraldehyde (pH 7.4) and were postfixed with $1 \%$ osmium tetroxide, after which they were conventionally prepared for transmission electron microscopy (H-800, Hitachi, Tokyo, Japan). We performed a morphometric analysis under an electron microscope using the method described previously [18]. with some modification. In brief, a uniform sampling of electron micrographs was used for the morphometric assay of each group. Ten random fields micrographed at $6,000 \times$ from each of three blocks were printed at a final magnification of $15,000 \times$ and analyzed on composite grids as described previously to calculate the density of autophagic vacuoles (number/100 $\mu^{2}$ ), i.e., both autophagosomes and autolysosomes, within a cardiomyocyte.

Statistical Analysis

All data are expressed as mean \pm SD. Statistical power analysis revealed that a sample size of $n=8$ would provide sufficient power $(0.8)$ to detect a difference between mean infarct size indices of $15 \%(\mathrm{SD}=9, \alpha=0.05)$. A group size of $\mathrm{n}=4$ was used for Western blot to provide a power of 0.8 to detect a difference between means of $20 \%(\mathrm{SD}=10, \alpha=0.05)$. Hemodynamic data were tested for normal distribution and subsequently analyzed by a twofactor repeated-measures analysis of variance for time and treatment. If an overall difference between the variables was observed, comparisons were performed as one-way ANOVA followed by Tukey's post-hoc test for inter-group differences and by Dunnett's for intragroup differences with baseline values as the reference time point. Analyses of infarct size and Western blot were performed using one-way ANOVA followed by Student's t-test with Bonferroni's correction for multiple comparisons to avoid type I error. For changes within 
and between groups a two-tailed $\mathrm{p}$ value less than 0.05 was considered significant in advance. (SPSS17 for Windows, SPSS Japan, Tokyo, Japan). 


\section{Results}

Of a total of 66, one heart in SEVO+MPG was not used secondary to intractable ventricular fibrillation after reperfusion and one heart was not used due to aortic rupture. Additional hearts were studied until each group had $n=8$ successful experiments for the infarct size study. There was no significant difference in body weight among groups. The concentration of sevoflurane in the coronary perfusate after $10 \mathrm{~min}$ of exposure was $0.25 \pm 0.02 \mathrm{mM}$. Sevoflurane was not detected in the effluent during the baseline, ischemic, and reperfusion periods.

\section{Hemodynamics}

Hemodynamic data are shown in Table 1. Baseline LVDP and CF were similar among the 4 groups. Treatment of sevoflurane and/or MPG did not significantly change LVDP, LVEDP and CF before ischemia. Recovery of LVDP was significantly greater in SEVO, MPG and SEVO+MPG compared with CTL throughout reperfusion period. After 120 min reperfusion, SEVO had significantly lower LVEDP compared to other groups. There was no significant difference in $\mathrm{CF}$ among all the groups throughout the experiment. This suggests that changes in CF could not account for the improved contractile recovery of the SEVO, MPG and SEVO+MPG groups.

Myocardial Infarct Size

Myocardial infarct size data are shown in Figure 2. Myocardial infarct size in SEVO was significantly reduced by approximately 50\% compared with CTL hearts (SEVO: $25 \pm 8 \%$ vs. CTL: $48 \pm 6 \%, \mathrm{p}<0.05)$. This cardioprotection achieved by sevoflurane was abolished by 
MPG (SEVO: $25 \pm 8 \%$ vs. SEVO+MPG: $36 \pm 10 \%, \mathrm{p}<0.05$ ). Treatment with MPG alone decreased the infarct size compared with CTL (MPG: $39 \pm 8 \%$ vs. CTL: $48 \pm 6 \%$, p<0.05).

\section{Electron Microscopy}

Figure 3 shows representative electron microphotographs of each study group. Although autophagic vacuoles were abundantly seen in cardiomyocytes from the untreated control heart (panel A: CTL), typical autophagosomes containing intracellular organelles, such as mitochondria and membrane-like structures (arrows), were even more increased after sevoflurane administration (panel B: SEVO). Autophagosomes were apparently diminished by addition of MPG (panels C and D: MPG and SEVO+MPG). Morphometric analysis of autophagosome density confirmed the above findings (Figure 3E).

Western Blot analysis

LC3-I, II, total AMPK and the phosphorylation state of AMPK after treatment with sevoflurane and MPG are illustrated by a representative Western blot in Figure 4A. In hearts pretreated with sevoflurane, myocardial expression of LC3-II was increased compared with control hearts. This increased expression of LC3-II was abolished by administration of MPG (Figure 4B). The ratio of phospho AMPK to total AMPK was significantly increased in SEVO compared with CTL (Figure 4C). This increase was not caused by unequal loading of the western blot, as shown by the detection of $\alpha$-tubulin. The combination of sevoflurane and MPG abolished this increased expression in SEVO. 


\section{Discussion}

This study demonstrated that ROS produced during sevoflurane exposure play a role in sevoflurane-induced cardioprotection through autophagy. Growing evidence suggests that ROS and autophagy are involved in cardioprotection by ischemic and volatile anesthetics preconditioning. The present study for the first time showed between link of these two mediators in sevoflurane-induced cardioprotection.

It was well documented that a burst ROS formation during the very early reperfusion period after myocardial ischemia-reperfusion causes serious damage to cardiomyocytes [19]. Scavenging ROS has been shown to attenuate myocardial reperfusion injury [20]. This is consistent with the present study demonstrating that treatment with MPG alone before ischemia reduced infarct size compared with control. MPG is a highly diffusible low molecular weight compound that scavenges superoxide and hydroxyl radicals produced both intra- and extracellularly $[21,22]$. In contrast, a low level of ROS was reported to function as a protective second messenger during ischemic preconditioning [23]. It has been shown that APC by sevoflurane is triggered by production of ROS during the preconditioning period [24]. In turn, this generation of ROS reduces the burst production of ROS during the very early reperfusion period, which results in reduction of myocardial infarct size. In the present study, administration of MPG to sevoflurane-treated hearts abolished the infarct size limiting effect of sevoflurane preconditioning. This suggests that production of ROS during sevoflurane exposure plays an important role in sevoflurane preconditioning.

There were no significant differences in LVDP and LVEDP among groups at 120 min of reperfusion (e.g. SEVO vs. MPG or SEVO+MPG) despite the difference in infarct size. A possible reason for this discrepancy may be due to stunning caused by global 
ischemia in a much greater area than the infarcted area of LV. Cohen et al. demonstrated that the enhanced functional recovery resulting from infarct size reduction by preconditioning becomes apparent 1 to 3 days later because of stunning [25].

Autophagy is a bulk degradation process in which cytosolic proteins and organelles are degraded through lysosomes. This is an evolutionally conserved process crucial for normal tissue homeostasis. Accumulating evidence suggests that autophagy is stimulated by ischemia and actually contributes to cardiomyocyte survival [26,27]. A recent study has shown that autophagy induced by ischemic preconditioning is essential for cardioprotection [28]. McCormick et al. demonstrated that enhancing autophagy confers protection against ischemia-reperfusion injury in cardiomyocytes [29]. Using in vivo swine models, SalaMercado et al. showed that induction of autophagy by chloramphenicol succinate before ischemia limited infarct size [14]. However, it is not known whether this upregulation of autophagy is mediated by ROS produced during sevoflurane exposure. Recently, Dai et al. demonstrated that mitochondrial oxidative stress mediates induction of autophagy in angiotensin-II treated mouse hearts [30]. Hariharan et al. have shown that oxidative stress stimulates autophagic flux during myocardial ischemia-reperfusion [31]. In the present study, electron microscopy demonstrated that there were more abundant autophagosomes in sevoflurane-treated hearts than control. This upregulation of autophagy was abolished by MPG. Furthermore, western blot analysis showed that the ratio of LC3 II/I (a marker of autophagy) and phosphorylation of AMPK were increased in sevoflurane preconditioning, which was abolished by MPG. Both LC3 and AMPK are key enzymes for formation of autophagosomes. Cardiac AMPK activation has been shown to be linked to inhibition of GSK3 $\beta$ [32] which is a critical mediator of cardioprotection by APC [5]. This suggests that 
ROS produced during sevoflurane exposure induced upregulation of autophagy in sevoflurane preconditioning.

The following study limitations should be acknowledged. The isolated Langendorff perfused heart perfusion may not completely reflect the conditions in vivo. However, the ex vivo conditions of the present study were designed to exclude possible confounding systemic hemodynamic and humoral effects of sevoflurane. Although we did not directly measure ROS during sevoflurane exposure, the dose $(1 \mathrm{mM})$ of MPG used in the present study has been shown to abolish the infarct size limiting effect of sevoflurane preconditioning [10].

In conclusion, sevoflurane exerts a cardioprotection against ischemia-reperfusion injury by ROS-mediated upregulation of autophagy. A more thorough understanding of the multiple signaling steps and the ultimate cytoprotective mechanisms underlying APC may lead to improvements in the management of patients at risk during perioperative periods. 


\section{Acknowledgments}

We thank Akiko Tsujimoto (Gifu University Graduate School of Medicine) for technical assistance. This study was conducted at the Laboratory Animal Facilities and Dental Bioscience I, Institute of Dental Research, Osaka Dental University, and was supported by Grant-in-Aid for Scientific Research (C) 23593008 from the Ministry of Education, Culture, Sports, Science and Technology of Japan (Masami Miyamae) (Tokyo, Japan). 


\section{REFERENCES}

1. Kersten JR, Schmeling TJ, Pagel PS, Gross GJ, Warltier DC. Isoflurane mimics ischemic preconditioning via activation of K(ATP) channels: reduction of myocardial infarct size with an acute memory phase. Anesthesiology. 1997;87:361-70.

2. Kaneda K, Miyamae M, Sugioka S, Okusa C, Inamura Y, Domae N, Kotani J, Figueredo VM. Sevoflurane enhances ethanol-induced cardiac preconditioning through modulation of protein kinase $\mathrm{C}$, mitochondrial KATP channels, and nitric oxide synthase, in guinea pig hearts. Anesth Analg. 2008;106:9-16.

3. Inamura Y, Miyamae M, Sugioka S, Kaneda K, Okusa C, Onishi A, Domae N, Kotani J, Figueredo VM. Aprotinin abolishes sevoflurane postconditioning by inhibiting nitric oxide production and phosphorylation of protein kinase $\mathrm{C}-\delta$ and glycogen synthase kinase 3ß. Anesthesiology. 2009;111:1036-43.

4. Inamura Y, Miyamae M, Sugioka S, Domae N, Kotani J. Sevoflurane postconditioning prevents activation of caspase 3 and 9 through antiapoptotic signaling after myocardial ischemia-reperfusion. J Anesth. 2010;24:215-24.

5. Onishi A, Miyamae M, Kaneda K, Kotani J, Figueredo VM. Direct evidence for inhibition of mitochondrial permeability transition pore opening by sevoflurane preconditioning in cardiomyocytes: Comparison with cyclosporine A. Eur J Pharmacol. 2012;675:40-6.

6. Julier K, da Silva R, Garcia C, Bestmann L, Frascarolo P, Zollinger A, Chassot PG, Schmid ER, Turina MI, von Segesser LK, Pasch T, Spahn DR, Zaugg M. Preconditioning by sevoflurane decreases biochemical markers for myocardial and renal dysfunction in coronary artery bypass graft surgery: a double-blinded, placebocontrolled, multicenter study. Anesthesiology. 2003;98:1315-27. 
7. Murry CE, Jennings RB, Reimer KA. Preconditioning with ischemia: a delay of lethal cell injury in ischemic myocardium. Circulation. 1986;74:1124-36.

8. Stadnicka A, Marinovic J, Ljubkovic M, Bienengraeber MW, Bosnjak ZJ. Volatile anesthetic-induced cardiac preconditioning. J Anesth. 2007;21:212-9.

9. Novalija E, Varadarajan SG, Camara AK, An J, Chen Q, Riess ML, Hogg N, Stowe DF. Anesthetic preconditioning: triggering role of reactive oxygen and nitrogen species in isolated hearts. Am J Physiol Heart Circ Physiol. 2002;283:H44-52.

10. Lamberts RR, Onderwater G, Hamdani N, Vreden MJ, Steenhuisen J, Eringa EC, Loer SA, Stienen GJ, Bouwman RA. Reactive oxygen species-induced stimulation of 5'AMP-activated protein kinase mediates sevoflurane-induced cardioprotection. Circulation. 2009;120:S10-5.

11. Klionsky DJ, Emr SD. Autophagy as a regulated pathway of cellular degradation. Science. 2000;290:1717-21.

12. Matsui Y, Takagi H, Qu X, Abdellatif M, Sakoda H, Asano T, Levine B, Sadoshima J. Distinct roles of autophagy in the heart during ischemia and reperfusion: roles of AMP-activated protein kinase and Beclin 1 in mediating autophagy. Circ Res. 2007;100:914-22.

13. Levine B, Kroemer G. Autophagy in the pathogenesis of disease. Cell. 2008;132:2742.

14. Sala-Mercado JA, Wider J, Undyala VV, Jahania S, Yoo W, Mentzer RM, Jr., Gottlieb RA, Przyklenk K. Profound cardioprotection with chloramphenicol succinate in the swine model of myocardial ischemia-reperfusion injury. Circulation. 2010;122:S179-84. 
15. Qiao S, Xie H, Wang C, Wu X, Liu H, Liu C. Delayed anesthetic preconditioning protects against myocardial infarction via activation of nuclear factor-kappaB and upregulation of autophagy. J Anesth. 2013;27:251-60.

16. Becalski A, Forsyth D, Casey V, Lau BP, Pepper K, Seaman S. Development and validation of a headspace method for determination of furan in food. Food Addit Contam. 2005;22:535-40.

17. Fishbein MC, Meerbaum S, Rit J, Lando U, Kanmatsuse K, Mercier JC, Corday E, Ganz W. Early phase acute myocardial infarct size quantification: validation of the triphenyl tetrazolium chloride tissue enzyme staining technique. Am Heart J. 1981;101:593-600.

18. Nakagawa M, Takemura G, Kanamori H, Goto K, Maruyama R, Tsujimoto A, Ohno T, Okada H, Ogino A, Esaki M, Miyata S, Li L, Ushikoshi H, Aoyama T, Kawasaki M, Nagashima K, Fujiwara T, Minatoguchi S, Fujiwara H. Mechanisms by which late coronary reperfusion mitigates postinfarction cardiac remodeling. Circ Res. 2008;103:98-106.

19. Hammond B, Hess ML. The oxygen free radical system: potential mediator of myocardial injury. J Am Coll Cardiol. 1985;6:215-20.

20. Bolli R, Jeroudi MO, Patel BS, Aruoma OI, Halliwell B, Lai EK, McCay PB. Marked reduction of free radical generation and contractile dysfunction by antioxidant therapy begun at the time of reperfusion. Evidence that myocardial "stunning" is a manifestation of reperfusion injury. Circ Res. 1989;65:607-22.

21. Mitsos SE, Fantone JC, Gallagher KP, Walden KM, Simpson PJ, Abrams GD, Schork MA, Lucchesi BR. Canine myocardial reperfusion injury: protection by a free radical scavenger, N-2-mercaptopropionyl glycine. J Cardiovasc Pharmacol. 1986;8:978-88. 
22. Miyamae M, Fujiwara H, Tanaka M, Yokota R, Takemura G, Itoh S, Domae N, Figueredo VM. Oxygen radicals mediate ultrastructural and metabolic protection of preconditioning in vivo in pig hearts. Exp Clin Cardiol. 2002;7:173-9.

23. Das DK, Maulik N, Sato M, Ray PS. Reactive oxygen species function as second messenger during ischemic preconditioning of heart. Mol Cell Biochem. 1999;196:59-67.

24. Bouwman RA, Musters RJ, van Beek-Harmsen BJ, de Lange JJ, Boer C. Reactive oxygen species precede protein kinase $\mathrm{C}$-delta activation independent of adenosine triphosphate-sensitive mitochondrial channel opening in sevoflurane-induced cardioprotection. Anesthesiology. 2004;100:506-14.

25. Cohen MV, Yang XM, Downey JM. Smaller infarct after preconditioning does not predict extent of early functional improvement of reperfused heart. Am J Physiol. 1999;277:H1754-H1761.

26. Yan L, Vatner DE, Kim SJ, Ge H, Masurekar M, Massover WH, Yang G, Matsui Y, Sadoshima J, Vatner SF. Autophagy in chronically ischemic myocardium. Proc Natl Acad Sci U S A. 2005;102:13807-12.

27. Kanamori H, Takemura G, Goto K, Maruyama R, Ono K, Nagao K, Tsujimoto A, Ogino A, Takeyama T, Kawaguchi T, Watanabe T, Kawasaki M, Fujiwara T, Fujiwara H, Seishima M, Minatoguchi S. Autophagy limits acute myocardial infarction induced by permanent coronary artery occlusion. Am J Physiol Heart Circ Physiol. 2011;300:H2261-71.

28. Huang C, Yitzhaki S, Perry CN, Liu W, Giricz Z, Mentzer RM, Jr., Gottlieb RA. Autophagy induced by ischemic preconditioning is essential for cardioprotection. $\mathbf{J}$ Cardiovasc Transl Res. 2010;3:365-73. 
29. McCormick J, Suleman N, Scarabelli TM, Knight RA, Latchman DS, Stephanou A. STAT1 deficiency in the heart protects against myocardial infarction by enhancing autophagy. J Cell Mol Med. 2012;16:386-93.

30. Dai DF, Rabinovitch P. Mitochondrial oxidative stress mediates induction of autophagy and hypertrophy in angiotensin-II treated mouse hearts. Autophagy. 2011;7:917-8.

31. Hariharan N, Zhai P, Sadoshima J. Oxidative stress stimulates autophagic flux during ischemia/reperfusion. Antioxid Redox Signal. 2011;14:2179-90.

32. Depre C, Wang L, Sui X, Qiu H, Hong C, Hedhli N, Ginion A, Shah A, Pelat M, Bertrand L, Wagner T, Gaussin V, Vatner SF. H11 kinase prevents myocardial infarction by preemptive preconditioning of the heart. Circ Res. 2006;98:280-8. 


\section{Figure Legends}

Figure 1: Schematic illustration of the experimental protocol. There were 4 groups. After 20 min baseline period, isolated perfused hearts were subjected to $30 \mathrm{~min}$ global ischemia and 120 min reperfusion. Anesthetic preconditioning was elicited by administration of $10 \mathrm{~min}$ of SEVO (2\%) with 10 min washout before 30 min ischemia. MPG was administered starting $30 \mathrm{~min}$ before ischemia in hearts from SEVO-treated or non-SEVO-treated animals. Tissue samples were obtained after reperfusion. CTL, control; MPG, n-(2-mercaptopropionyl) glycine; SEVO, sevoflurane.

Figure 2: Infarct size as a percentage of left ventricle in 4 groups. After 120min reperfusion, infarct size was significantly reduced in SEVO compared to CTL. This cardioprotection of SEVO was abolished by MPG. MPG alone decreased infarct size compared to CTL. Data are presented as mean $\pm \mathrm{SD} ; * \mathrm{p}<0.05$ versus CTL; $\uparrow \mathrm{p}<0.05$ versus MPG and SEVO+MPG. CTL, control; MPG, n-(2-mercaptopropionyl) glycine; SEVO, sevoflurane (n=8 for each group).

Figure 3: Electron microphotographs of cardiomyocytes. Autophagic vacuoles were seen in cardiomyocytes from the untreated control (panel A). Typical autophagosomes containing intracellular organelles, such as mitochondria and membrane-like structures (arrows), were more increased after sevoflurane administration (panel B: SEVO). Autophagosomes were apparently diminished by addition of MPG (panels C and D: MPG and SEVO+MPG). (E) Graph showing the density of autophagic vacuoles (number/100 $\mu \mathrm{m}^{2}$ ) within a cardiomyocyte in each group. ${ }^{\dagger} \mathrm{p}<0.05$ versus other groups. Nucl, nucleus. Bars, $1 \mu \mathrm{m}$. CTL, control; MPG, n-(2-mercaptopropionyl) glycine; SEVO, sevoflurane 
Figure 4: (A) Representative western blot analysis of LC3-I, II, phosphorylated AMPK and total AMPK from left ventricular samples obtained after 120 min of reperfusion. (B) The ratio of LC3-II to LC3-I (LC3-II/I) was increased in SEVO compared to CTL. Administration of MPG abolished this increased LC3-II/I in the SEVO group. ${ }^{*} \mathrm{p}<0.05$ versus CTL. ${ }^{\dagger} \mathrm{p}<0.05$ versus MPG and SEVO+MPG. (C) Densitometric evaluation of four experiments as the $x$-fold increase in average light density $v s$. CTL. The results are presented as the ratio of the phosphorylated AMPK to total AMPK. The average light intensity was multiplied by 100 to facilitate the presentation of an $\mathrm{x}$-fold increase. Data are presented as mean \pm standard deviation. ${ }^{*} \mathrm{p}<0.05$ versus CTL. ${ }^{\dagger} \mathrm{p}<0.05$ versus MPG and SEVO+MPG. AMPK, 5'AMP-activated protein kinase; CTL, control; LC3-I, II, microtubule-associated protein light chain I and II; MPG, n-(2-mercaptopropionyl) glycine; SEVO, sevoflurane. 
Figure 1

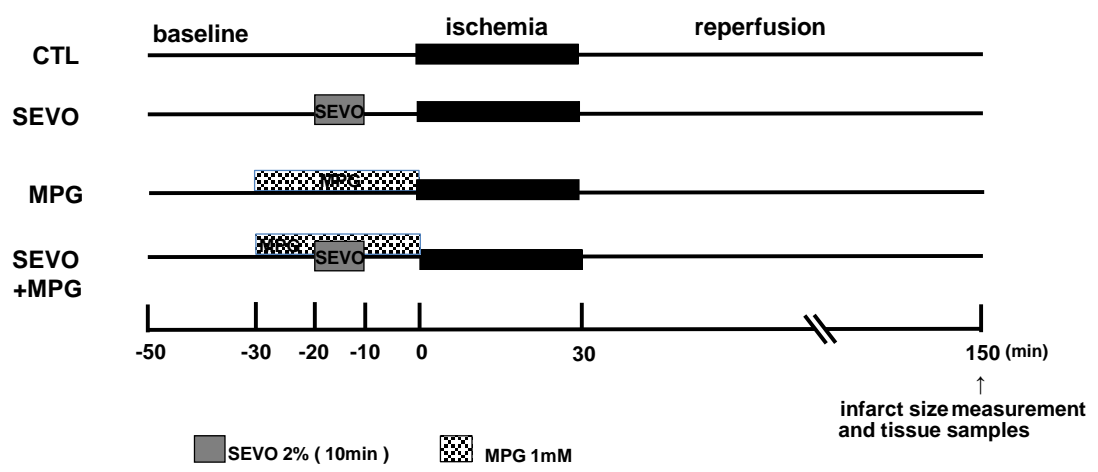


Figure 2

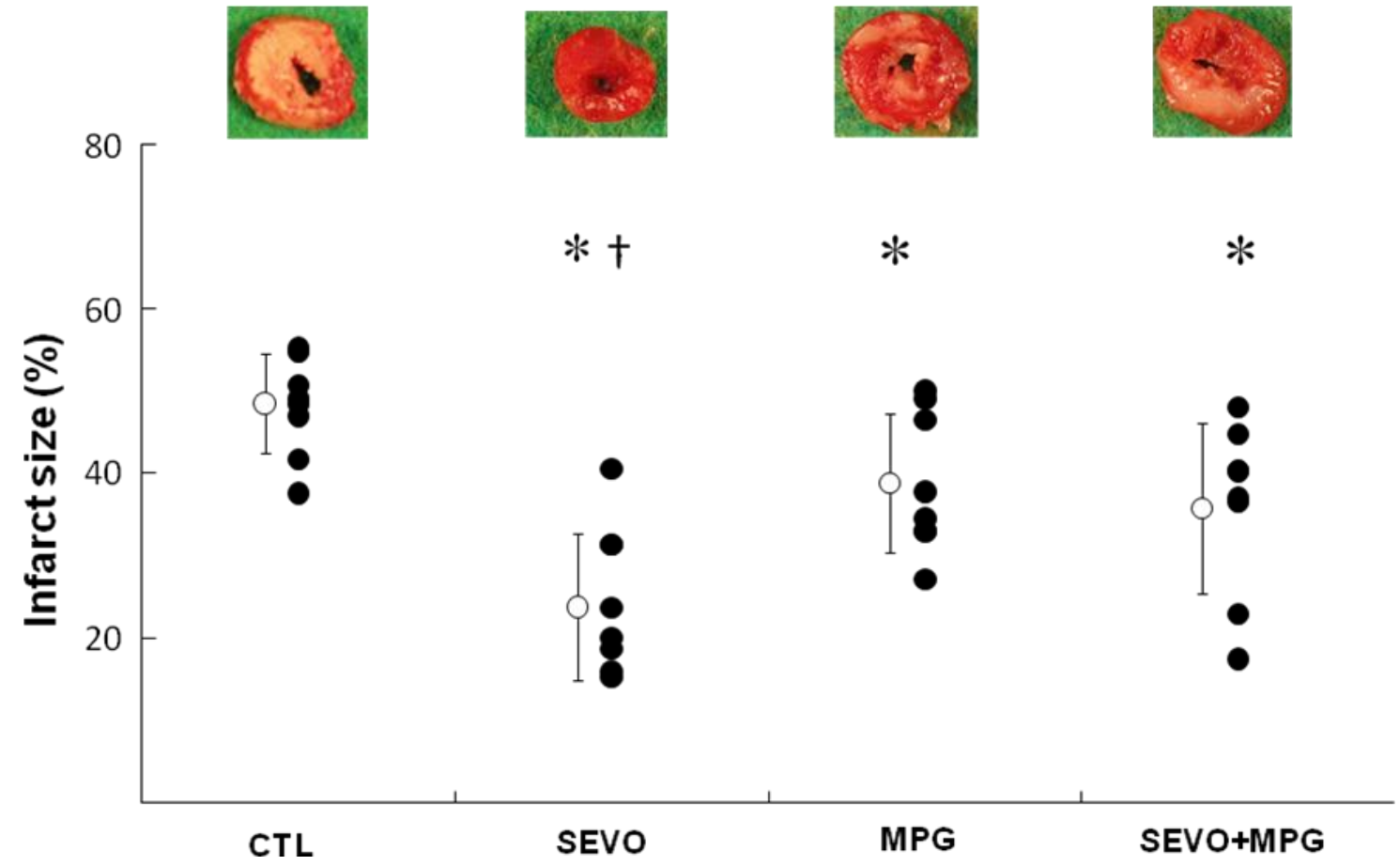


Figure 3
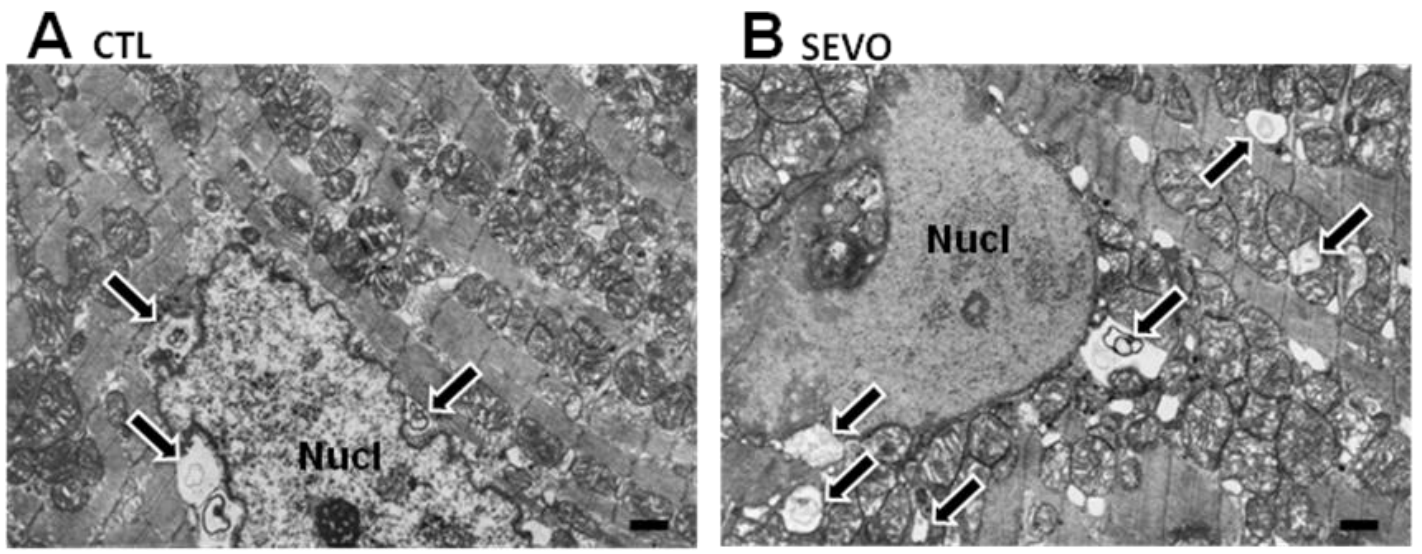

\section{MPG}

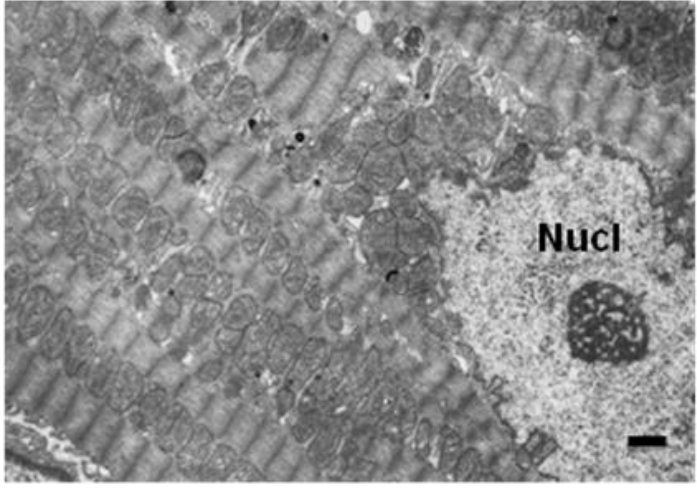

D SEVO+MPG

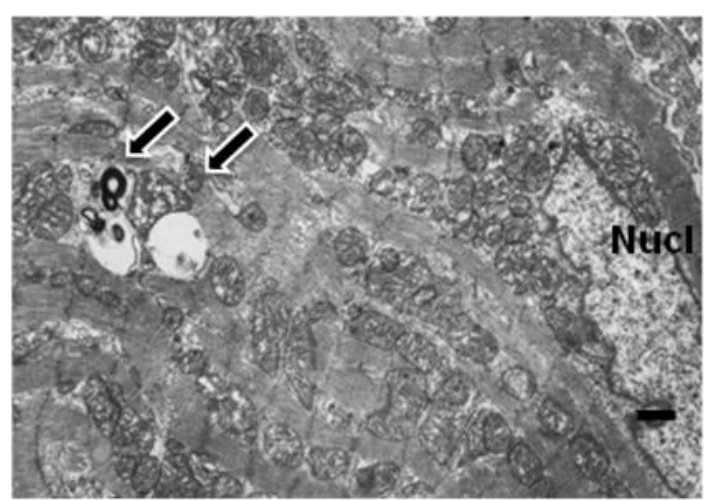

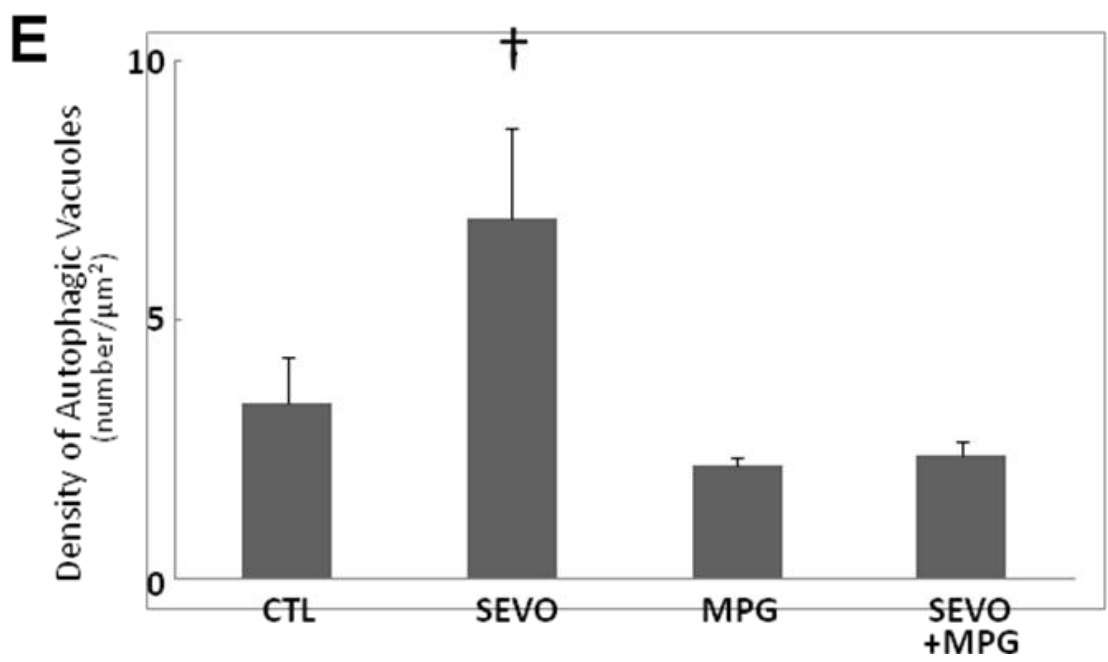


Figure 4

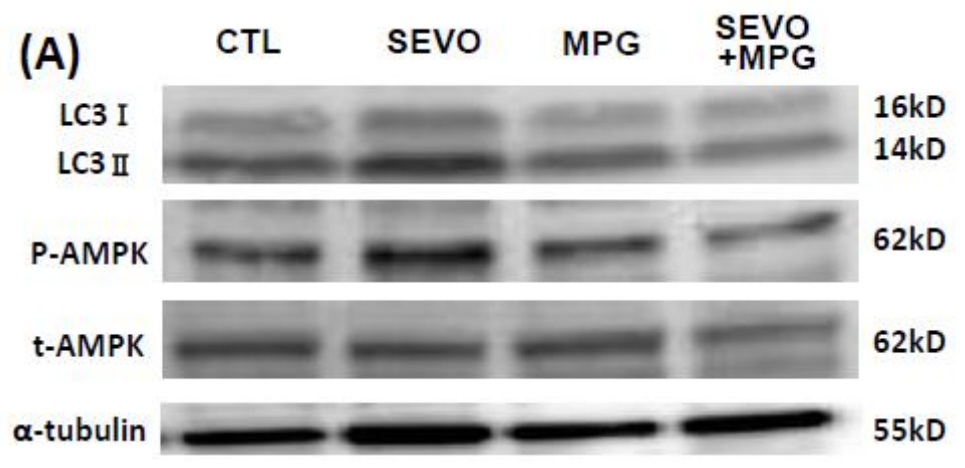

(B)

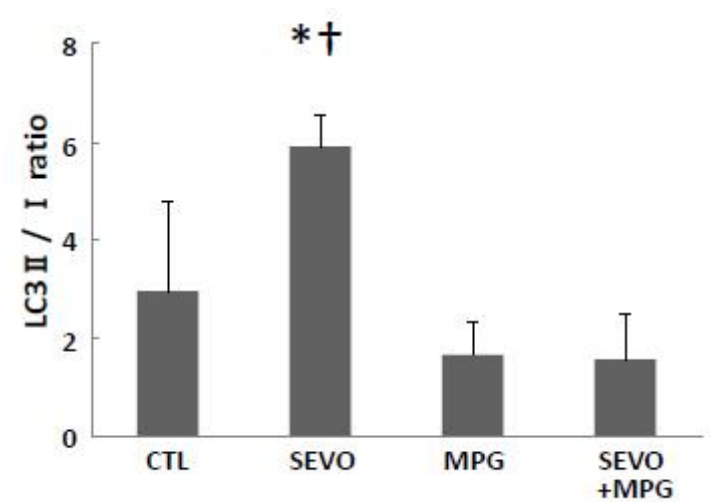

(C)

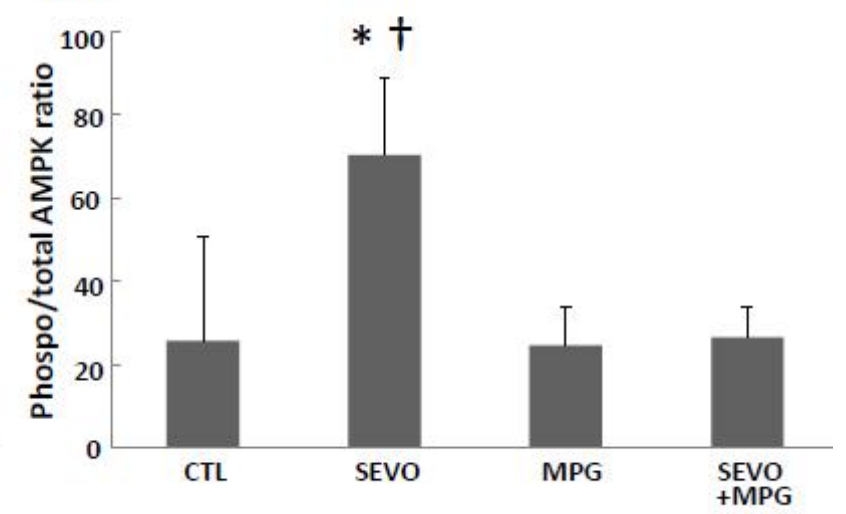


Table 1

TABLE 1. Hemodynamic Variables

\begin{tabular}{|c|c|c|c|c|c|c|c|c|c|c|c|c|c|c|}
\hline & \multirow{3}{*}{ Baseline } & \multirow{2}{*}{\multicolumn{3}{|c|}{$\begin{array}{c}\text { After } \\
\text { treatment } \\
(\text { SEVO/MPG) } \\
\end{array}$}} & \multirow{2}{*}{$\begin{array}{l}\text { Before } \\
\text { ischemia }\end{array}$} & \multicolumn{9}{|c|}{ Reperfusion (min) } \\
\hline & & & & & & \multicolumn{3}{|c|}{30} & \multicolumn{3}{|c|}{60} & \multicolumn{3}{|c|}{120} \\
\hline \multicolumn{14}{|l|}{ LVDP (mmHg) } & \\
\hline CTL & $113 \pm 15$ & 117 & \pm & 13 & $116 \pm 13$ & 39 & \pm & 7 & 34 & \pm & 9 & 26 & \pm & 6 \\
\hline SEVO & $101 \pm 13$ & 100 & \pm & 12 & $103 \pm 12$ & 63 & \pm & $7 *$ & 60 & \pm & $8^{*}$ & 52 & \pm & $8^{*}$ \\
\hline MPG & $113 \pm 22$ & 107 & \pm & 23 & $104 \pm 24$ & 46 & \pm & $19 *$ & 48 & \pm & $15^{*}$ & 48 & \pm & $9 *$ \\
\hline SEVO+MPG & $102 \pm 17$ & 108 & \pm & 21 & $110 \pm 21$ & 51 & \pm & $18^{*}$ & 50 & \pm & $17 *$ & 46 & & $16^{*}$ \\
\hline \multicolumn{15}{|l|}{ LVEDP(mmHg) } \\
\hline CTL & $10 \pm 0$ & 11 & \pm & 2 & $11 \pm 2$ & 50 & \pm & 10 & 55 & \pm & 15 & 57 & \pm & 15 \\
\hline SEVO & $10 \pm 0$ & 10 & \pm & 3 & $10 \pm 2$ & 24 & \pm & $7 *$ & 23 & \pm & $4 *$ & 23 & \pm & $5^{*}$ \\
\hline MPG & $10 \pm 0$ & 11 & \pm & 7 & $11 \pm 7$ & 48 & \pm & 26 & 48 & \pm & 25 & 40 & & 26 \\
\hline SEVO+MPG & $10 \pm 0$ & 6 & \pm & 6 & $6 \pm 6$ & 39 & \pm & 16 & 36 & \pm & 16 & 35 & \pm & $18^{*}$ \\
\hline \multicolumn{15}{|l|}{$\mathrm{CF}(\mathrm{mL} / \mathrm{min})$} \\
\hline CTL & $27 \pm 6$ & 28 & \pm & 6 & $28 \pm 6$ & 20 & \pm & 4 & 19 & \pm & 4 & 18 & \pm & 4 \\
\hline SEVO & $32 \pm 8$ & 28 & \pm & 5 & $27 \pm 5$ & 22 & \pm & 4 & 21 & \pm & 5 & 21 & \pm & 5 \\
\hline MPG & $30 \pm 9$ & 30 & \pm & 9 & $29 \pm 8$ & 22 & \pm & 6 & 20 & \pm & 6 & 19 & \pm & 7 \\
\hline SEVO+MPG & $26 \pm 5$ & 26 & \pm & 5 & $27 \pm 4$ & 21 & \pm & 7 & 18 & \pm & 6 & 16 & \pm & 6 \\
\hline \multicolumn{15}{|c|}{$\begin{array}{l}\text { Data are presented as mean } \pm \text { SD. LVDP=left ventricular developed pressure; LVEDP=left ventricular end- } \\
\text { diastolic pressure; } \quad \mathrm{CF}=\text { coronary flow; } \quad \mathrm{CTL}=\text { control; } \mathrm{MPG}=\mathrm{N}-2 \text {-mercaptopropionyl glycine; } \\
\text { SEVO=sevoflurane;. *p<0.05 vs. CTL, } \mathrm{n}=8 \text { for each group. }\end{array}$} \\
\hline
\end{tabular}

